\title{
Optical transitions and excitonic recombination in InAs/InP self-assembled quantum wires
}

\author{
Benito Alén, ${ }^{\text {a) }}$ Juan Martínez-Pastor, and Alberto García-Cristobal \\ Instituto de Ciencia de Materiales, Universidad de Valencia, P.O. Box 2085, 46071 Valencia, Spain \\ Luisa González and Jorge M. García \\ Instituto de Microelectrónica de Madrid (CNM-CSIC), Isaac Newton 8, 28760 Tres Cantos (Madrid), Spain
}

(Received 18 December 2000; accepted for publication 25 April 2001)

\begin{abstract}
InAs self-assembled quantum wire structures have been grown on InP substrates and studied by means of photoluminescence and polarized-light absorption measurements. According to our calculations, the observed optical transitions in each sample are consistent with wires of different heights, namely from 6 to 13 monolayers. The nonradiative mechanism limiting the emission intensity at room temperature is related to thermal escape of carriers out of the wires. (c) 2001 American Institute of Physics. [DOI: 10.1063/1.1379991]
\end{abstract}

The study of self-organized quantum nanostructures of InAs grown on $\mathrm{InP}(001)$ have received considerable attention in recent years due to their potentiality for optoelectronic devices operating in the wavelength range $1.3-1.55$ $\mu \mathrm{m} .{ }^{1-4}$ Recent works have demonstrated that self-assembled quantum dot $(\mathrm{QD})$ and quantum wire (QWR) structures can be obtained by molecular-beam epitaxy (MBE) growth of InAs on InP, ${ }^{2-6}$ above a certain critical thickness. The reflection high energy diffraction pattern shows that growth is twodimensional (2D) mode-like during the InAs deposition and 2D/three-dimensional (3D) mode transition occurs after growth interruption. ${ }^{4}$

In this work, we report photoluminiscence (PL) studies on several samples containing self-assembled InAs/InP QWRs. Linear polarization dependent transmission experiments on thinned samples have been carried out in order to corroborate the polarization anisotropy observed in the emission of these QWRs, even at short wavelength excitation. ${ }^{4}$ The optical transitions observed in PL and transmission spectra in several similar samples have been compared with calculated values, by means of an eigenfunction expansion method.

A 2.5 monolayer $(1 \mathrm{ML}=0.303 \mathrm{~nm})$ thick InAs layer was deposited after a $200 \mathrm{~nm}$ thick InP buffer layer by MBE at a substrate temperature $T_{S}=400^{\circ} \mathrm{C}$. After InAs deposition, growth is interrupted and $T_{S}$ is increased to $480^{\circ} \mathrm{C}$ for allowing InAs QWR self-assembling. Finally, a $50 \mathrm{~nm}$ thick InP capping layer was grown. Several samples with the same InAs coverage have been grown under similar conditions. The deposited amount of InAs, $2.5 \mathrm{ML}$, is a nominal value. Due to the As/P exchange at the $\mathrm{InP}$ surface exposed to As pressure, ${ }^{7}$ a larger amount of InAs could be involved in the InAs QWR formation. Atomic force microsocopy (AFM) on similar samples without capping has shown that QWR-like nanostructures appear oriented along the [1-10] direction. The average value of the wire height, $h$, is about $0.14 \mathrm{~nm}$ ( $\sim 5 \mathrm{ML})$ and a typical length exceeds $1 \mu \mathrm{m}$. The average width of the wires, $w$, is around $18 \mathrm{~nm}$ and the pitch period,

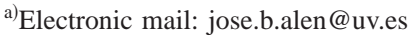

$\lambda \sim 24 \mathrm{~nm}$. Due to AFM experimental errors, these values are compatible with the nominal InAs coverage or even larger amounts, up to $4 \mathrm{ML}$, as just explained. More details about growth and structural properties can be found in Refs. 4 and 7.

The PL measurements have been carried out in the temperature range $30-300 \mathrm{~K}$. The excitation wavelength was $514.5 \mathrm{~nm}$ and incident power densities in the range $10^{3}-10^{4} \mathrm{~W} / \mathrm{cm}^{2}$. The PL signal was dispersed by a $1 / 8 \mathrm{~m}$ focal length double monochromator, and synchronously detected by an InGaAs photodiode. Linear polarization dependent transmission experiments were performed on $100 \mu \mathrm{m}$ thick samples. The white light of a halogen lamp was dispersed by a $0.5 \mathrm{~m}$ focal length single monochromator and linearly polarized parallel or perpendicular to the wire axis before being focused by a $20 \times$ microscope objective onto the sample surface under normal incidence conditions. The samples were oriented with the wire axis at $45^{\circ}$ with respect to the grating grooves for avoiding polarization effects from the system. The signal was synchronously detected by an InGaAs photodiode held below the sample inside the same flux liquid $\mathrm{N}_{2}$ cryostat.

Figure 1 shows the absorbance [Fig. 1(a)] and PL spectra [Fig. 1(b)] measured at $85 \mathrm{~K}$ in a typical sample containing self-assembled QWR structures. In both cases, several resonances are observed below the excitonic InP optical transition (around $1.42 \mathrm{eV}$ ). The absorbance of the quantum structures [Fig. 1(a)] is very weak, as compared to the InP band edge, in accordance with the small InAs thickness. The ratio between absorbance parallel $\left(A_{x}\right)$ and perpendicular $\left(A_{y}\right)$ to the wire axis $(x), A_{x} / A_{y}$ is around 2, which is also the approximate value found for emission. ${ }^{4}$ This high value can be only due to the wire-like geometry, strengthened by the simultaneous effect of different strain values between $x$ and $y$ directions. ${ }^{7,8}$ In this way, strong mixing in the hole subbands is expected, which would account for the anisotropy in the optical properties of our QWR structures. ${ }^{9,10}$

The characteristic emission of our samples is not a single broad Gaussian band, but instead several Gaussian components can be well resolved, as shown in Fig. 1(b) for sample 1. These components can be related to wires of different 


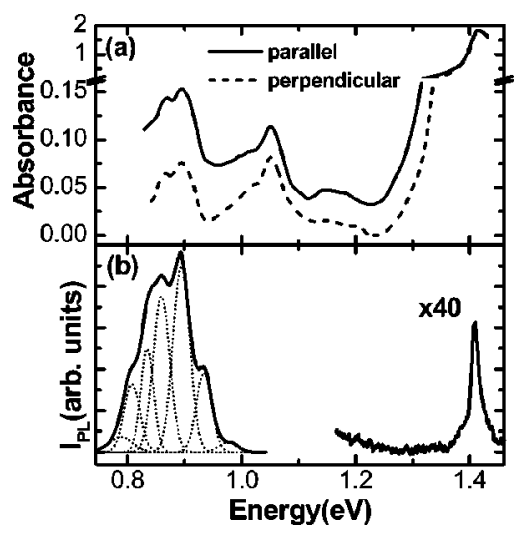

FIG. 1. Absorbance (a) and PL (b) spectra of sample 1 measured at $85 \mathrm{~K}$ are shown.

heights existing in the illuminated area, instead of excited states. This hypothesis is compatible with the fact that most of the components are observed under a large range of excitation densities $\left(10^{3}-10^{6} \mathrm{~W} / \mathrm{cm}^{2}\right)$ and temperatures $(30-300$ $\mathrm{K})$. The Gaussian linewidths of these components, $\sigma$, are typically in the range $15-35 \mathrm{meV}$, arising from the wire width fluctuations.

An eigenfunction expansion method ${ }^{11}$ has been used to obtain the single electron and hole states of an infinite array of coupled rectangular quantum wires $(w \sim 18 \mathrm{~nm}$ and $\lambda$ $\sim 24 \mathrm{~nm}$ ) biaxially strained (isotropic). No wetting layer (commonly present in self-assembled QDs) has been taken into account in the calculation, because PL and transmission experiments do not reveal its existence in our samples. More details of the model and calculations will be published elsewhere. The continuous line in Fig. 2 stands for the calculated values of band-to-band optical transitions as a function of the wire height. We do not know, a priori, the exact value of the wire height responsible of each PL Gaussian component. The starting point is the assumption that two consecutive PL components arise from wires with a height difference of 1 ML. ${ }^{1,3}$ The next step is to allocate the experimental PL peak energies of the different components ( $y$ coordinate of the experimental points on Fig. 2) adding a fixed number of monolayers, $N$, for building the $x$ coordinate. For $N=5$, we find a good correspondence with the calculated curve. On the one hand, this coincidence gives us confidence about the aforementioned assumption. On the other hand, we deduce that the wires responsible for the observed emission band appear to have heights from 7 to 13 MLs for samples 1 and 2 and 9 to 13 MLs for sample 3, in agreement with the $7 \mathrm{ML}$ height fluctuations between different wires measured from AFM micrographs taken on similar samples without capping. ${ }^{4}$ However, these absolute values for the wire heights should be taken as an estimate. On one side, the exciton binding energy could shift calculated values around $20 \mathrm{meV}$ towards lower energies. On the other side, if the anisotropy of the strain in our QWRs was incorporated in the calculation, the curve would shift to higher energies around $20 \mathrm{meV}^{12}$ More incertitude can arise from the choice of the band offset (70:30 have been chosen before strain for conduction to valence bands).

Most of the investigated samples are emitting light at around $1.5 \mu \mathrm{m}$ even at room temperature, which is an interDownloaded 16 Jun 2010 to 161.111 .235 .252 . Redistribution subje

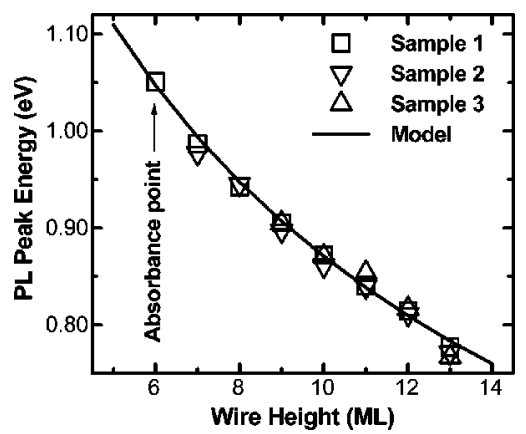

FIG. 2. Comparison between experimental data (symbols) and calculated values of band-to-band optical transitions in a coupled array of QWRs at $30 \mathrm{~K}$.

esting spectral region for optoelectronic devices. However, the overall PL integrated intensity is one to two orders of magnitude lower than that measured at $30 \mathrm{~K}$, as shown in Fig. 3 for sample 1. The investigation of the mechanisms responsible for the temperature quenching of the luminescence is then an important task to determine the potential application of these structures. The experimental temperature dependence of the PL integrated intensity (of the whole band and also for every component of the band) can be accounted for by a Boltzmann model for excitonic recombination, with two characteristic activation energies: ${ }^{13}$

$$
I_{\mathrm{PL}}(T)=\frac{I_{0}}{1+\tau_{0}\left[\Gamma_{1} \exp \left(-E_{1} / k T\right)+\Gamma_{2} \exp \left(-E_{2} / k T\right)\right]},
$$

where $I_{0}$ is the PL integrated intensity at $0 \mathrm{~K}, \tau_{0}$ the radiative recombination time, assumed to be independent of temperature (strong spatial localization effects on excitons), $E_{1}$ and $E_{2}$ are the activation energies of the two quenching mechanisms, and $\Gamma_{1}$ and $\Gamma_{2}$ the two related scattering rates.

The line in Fig. 3 is the best fitting curve to the experimental temperature dependence of the integrated intensity of the whole PL band (all components) for sample 1. The fitting parameters $\left(\tau_{0}=170 \mathrm{ps}, \quad \Gamma_{1}=7.5 \times 10^{10} \mathrm{~s}^{-1}, E_{1}=33 \mathrm{meV}\right.$, $\Gamma_{2}=2 \times 10^{16} \mathrm{~s}^{-1}$, and $E_{2}=270 \mathrm{meV}$ ) are thus representative

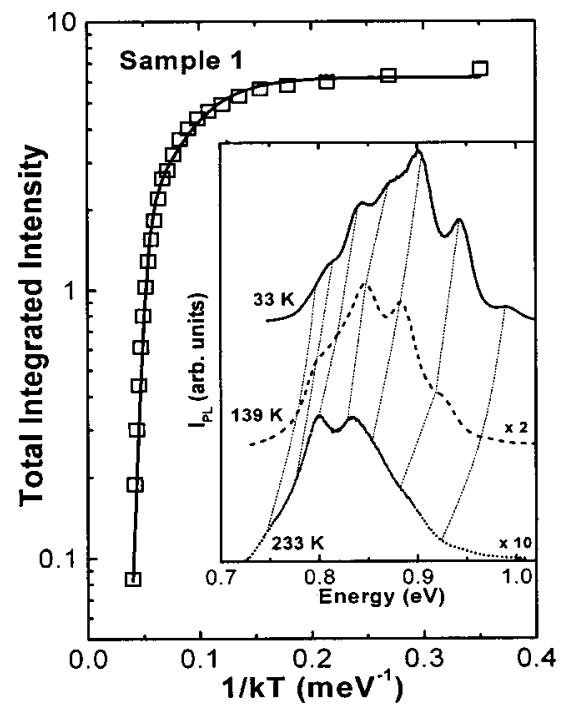

FIG. 3. Arrhenius plot of the PL integrated intensity of the whole emission band for sample 1 and fitting curve according to Eq. 1 is shown. The inset shows the PL spectra of the same sample at different temperatures.

to AIP license or copyright; see http://apl.aip.org/apl/copyright.jsp 


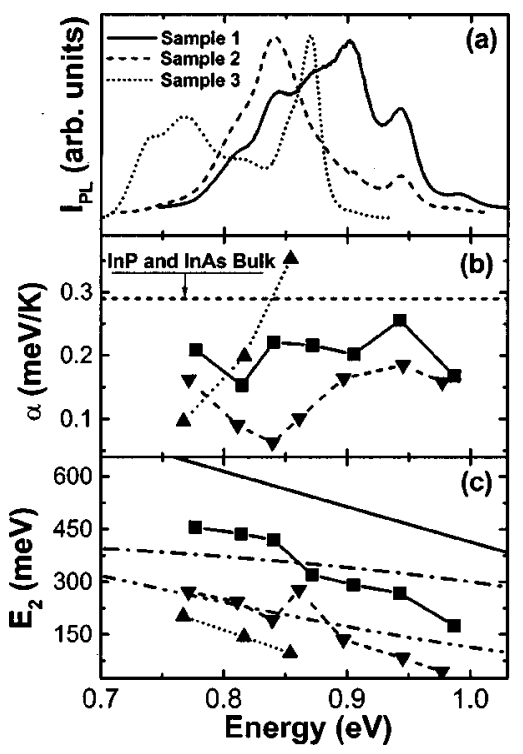

FIG. 4. Low-temperature PL spectra $(30 \mathrm{~K})$ of the different samples (a), temperature coefficient of the Gaussian peak energies (b) and activation energies (c) obtained from the temperature dependence of the integrated intensity for each Gaussian component are shown.

of the average behavior of the emission band, being the most important $\tau_{0}, E_{1}$, and $E_{2}$. The value of $E_{1}$ is similar in the other two samples (20-40 meV) and also for every PL components. This low activation energy is determining the lowtemperature quenching of the PL and it is usually associated to carrier recombination through impurities in the quantum structure or its interfaces with InP. In this way, it is difficult to establish some correlation with intrinsic parameters of the wires. On the contrary, $E_{2}$, is the activation energy associated with the high-temperature quenching of the PL band, and hence is the parameter to be associated to the intrinsic nonradiative recombination mechanism in self-assembled QWRs. In fact, we observe how high-energy Gaussian components (smaller wires) quench faster than the low-energy ones (taller wires), as shown in the inset of Fig. 3. Therefore, we can obtain more information about such nonradiative mechanism if we take account of the temperature dependence of the different Gaussian components.

In Fig. 4(a), we have plotted the PL spectra at $30 \mathrm{~K}$ of the three samples studied here, in order to correlate the different components with the data points in Figs. 4(b) and 4(c). The correlation with their corresponding wire heights can be done through Fig. 2. Firstly, we find that the temperature coefficients of the different peak energies are mostly between 0.1 and $0.2 \mathrm{meV} / \mathrm{K}$ as shown in Fig. 4(b). These low values, as compared to the bulk value for InAs and InP $(0.29 \mathrm{meV} /$ $\mathrm{K})$, could be related to the strong spatial localization of excitons in the QWR structures. From Fig. 4(c), we observe how the value of $E_{2}$ for the different Gaussian components in each sample decreases with increasing the confinement energy (decreasing the wire height), as expected from the quenching behavior shown in the inset of Fig. 3. Thermal escape of carriers, either ambipolar ${ }^{13}$ or unipolar, ${ }^{14}$ is known as the most important temperature dependent nonradiative mechanism in quantum wells, and it seems to be also the case in our QWRs. If the quenching mechanism was an ambipolar thermal escape of carriers (simultaneously electron+hole), the approximate escape energy would be that represented by the straight line in Fig. 4(c), taken as an upper limit. However, this limit is far from the low values measured in samples 2 and 3. In Fig. 4(c), we have also represented the unipolar thermal escape energies for holes (dot-dashed) and electrons (2-dot-dashed) deduced from our calculations. These curves are closer to the experimental values, even if a change from hole to electron escape would be needed for explaining the experimental values in sample 1. A more definite conclusion could be outdrawn if the decay times as a function of temperature for each PL component could be measured directly. The values obtained for $\tau_{0}$ by the Boltzmann model are in the range 1-10 ns for the Gaussian components below the central point of the emission (highest wires) in samples 1 and 2, and quickly decreases below 100 ps for high-energy PL Gaussian components (smallest wires), as expected in QWRs according to the increase of confinement. ${ }^{15}$

In conclusion, several optical transitions corresponding to wires of different heights have been measured in the different samples. An anisotropy ratio around a factor two is found in our QWR samples, either in absorption or emission, due to the wire geometry and strain anisotropy. The nonradiative mechanism responsible for the PL temperature quenching is related to the thermal escape of carriers out of the wires towards the barrier material, InP.

This work has been supported under project No. TIC991035-C02 of the Spanish CICYT.

${ }^{1}$ A. Rudra, R. Houdré, J. F. Carlin, and M. Ilegems, J. Cryst. Growth $\mathbf{1 3 6}$ 278 (1994).

${ }^{2}$ A. Ponchet, A. Le Corre, H. L'Haridon, B. Lambert, and S. Salaün, Appl. Phys. Lett. 67, 1850 (1995).

${ }^{3}$ J. Brault, M. Gendry, G. Grenet, G. Hollinger, Y. Desières, and T. Benyattou, Appl. Phys. Lett. 73, 2932 (1998).

${ }^{4}$ L. González, J. M. García, R. García, F. Briones, J. Martínez-Pastor, and C. Ballesteros, Appl. Phys. Lett. 76, 1104 (2000).

${ }^{5} \mathrm{H}$. Li, Q. Zhuang, X. Kong, Z. Wang, and T. Daniels-Race, J. Cryst. Growth 205, 613 (1999).

${ }^{6} \mathrm{C}$. Walther, W. Hoerstel, H. Niehus, J. Erxmeyer, and W. T. Masselink, J. Cryst. Growth 209, 572 (2000).

${ }^{7}$ J. M. García, L. González, M. U. González, J. P. Silveira, Y. González, and F. Briones (unpublished).

${ }^{8}$ J. A. Prieto, G. Armelles, C. Priester, J. M. García, L. González, and R. García, Appl. Phys. Lett. 76, 2197 (2000).

${ }^{9}$ O. Stier and D. Bimberg, Phys. Rev. B 55, 7726 (1997).

${ }^{10}$ M. Notomi, J. Hammersberg, J. Zeman, H. Weman, M. Potemski, H. Sugiura, and T. Tamamura, Phys. Rev. Lett. 80, 3125 (1998).

${ }^{11}$ G. A. Baraff and D. Gershoni, Phys. Rev. B 43, 4011 (1991).

${ }^{12}$ M. Notomi, J. Hammersberg, H. Weman, S. Nojima, H. Sugiura, M. Okamoto, T. Tamamura, and M. Potemski, Phys. Rev. B 52, 11147 (1995).

${ }^{13}$ E. M. Daly, T. J. Glynn, J. D. Lambkin, L. Considine, and S. Walsh, Phys. Rev. B 52, 4696 (1995).

${ }^{14}$ M. Gurioli, J. Martínez-Pastor, M. Colocci, C. Deparis, B. Chastaingt, and J. Massies, Phys. Rev. B 46, 6922 (1992).

${ }^{15}$ D. Gershoni, M. Katz, W. Wegscheider, L. N. Pfeiffer, R. A. Logan, and K. West, Phys. Rev. B 50, 8930 (1994). 